\title{
Investing in Instructors: Creating Intelligent Feedback Loops in Large Foun- dational Courses for Undergraduate Engineering
}

\section{Dr. Jacob R. Grohs, Virginia Tech}

Jacob Grohs is an Assistant Professor in Engineering Education at Virginia Tech with Affiliate Faculty status in Biomedical Engineering and Mechanics and the Learning Sciences and Technologies at Virginia Tech. He holds degrees in Engineering Mechanics (BS, MS) and in Educational Psychology (MAEd, $\mathrm{PhD})$.

\section{Dr. David B. Knight, Virginia Tech}

David Knight is Assistant Professor and Assistant Department Head for Graduate Programs in the Department of Engineering Education at Virginia Tech. He is also Director of International Engagement in Engineering Education and affiliate faculty with the Higher Education Program at Virginia Tech. His research tends to be at the macro-scale, focused on a systems-level perspective of how engineering education can become more effective, efficient, and inclusive.

\section{Ms. Michelle Soledad, Virginia Tech}

Michelle Soledad is a $\mathrm{PhD}$ candidate in the Department of Engineering Education at Virginia Tech. Her research interests include faculty development and data-informed reflective practice. Ms. Soledad has degrees in Electrical Engineering (BS, ME) from the Ateneo de Davao University (ADDU) in Davao City, Philippines, where she continues to be a faculty member of the Electrical Engineering Department. She also served as Department Chair and was a member of the University Research Council before pursuing doctoral studies. Prior to joining ADDU in 2008, Ms. Soledad was a Senior Team Lead for Accenture, where she worked on and managed systems maintenance and enhancement projects.

\section{Prof. Scott W Case, Virginia Tech}

Scott W. Case is the Reynolds Metals Professor of Engineering Mechanics at Virginia Tech. He has previously served as associate department head of Engineering Science and Mechanics and as Interim Associate Dean for Academic Affairs within the College of Engineering.

\section{Dr. Homero Gregorio Murzi, Virginia Tech}

Homero Murzi is an Associate Professor of Practice in the Department of Engineering Education at Virginia Tech. He holds degrees in Industrial Engineering (BS, MS), Master of Business Administration (MBA) and in Engineering Education (PhD). His research focuses on contemporary and inclusive pedagogical practices, environmental, ethics and humanitarian engineering, and non-traditional knowledge transfer. Homero has been recognized as a Fulbright scholar and was inducted in the Bouchet Honor Society.

\section{Natasha Smith, Virginia Tech}

Natasha is the Director of Enrollment Management for the College of Engineering as well as an undergraduate academic and career advisor for General Engineering students. These dual roles allow Natasha the unique opportunity to understand and articulate viewpoints of both administration and students.

Natasha strives to implement innovative and systematic technological advances to academic advising and enrollment management. 


\section{Investing in Instructors: Creating Intelligent Feedback Loops in Large Foundational Courses for Undergraduate Engineering}

\section{Introduction}

The drive to encourage young people to pursue degrees and careers in engineering has led to an increase in student populations in engineering programs. For some institutions, such as large public research institutions, this has led to large class sizes for courses that are commonly taken across multiple programs [1], [2]. While this decision is reasonable from an operational and resource management perspective, research on large classes have shown that students suffer decreased academic engagement, motivation and achievement [3]-[5] . Instructors, on the other hand, report having difficulty establishing rapport with their students and a growing inability to monitor students' learning gains and provide quality individualized feedback [4]-[6]. To address these issues, our project draws from Lattuca and Stark's Academic Plan model [9], which incorporates a thorough consideration of factors influencing curricular activities that can be applied at the course, program, and institutional levels, and assumes that instructors are key actors in curriculum development and revision [9]. We aim to revitalize feedback loops by productively leveraging institutional data to help instructors and departments continuously improve [10].

\section{Project overview}

A fundamental characteristic of the learning organization theory of change is the need to understand both the individual and systems level perspectives [11]. We prioritize regular engagement between faculty and institutional support structures to collaboratively identify problems and systematically establish continuous improvement, a key strength of this theory of change. We strive to build a learning organization ecosystem which will transform the undergraduate engineering educational environment by: (1) engaging, as equal partners, the faculty responsible for teaching large foundational engineering courses; (2) recreating the broken feedback loop from the Academic Plan Model by infusing data back into the decision-making processes of faculty and departments; and (3) collaboratively defining problems with faculty and administrators within the system and iteratively intervene.

This Exploration and Design Institutional and Community Transformation IUSE project will prompt and study the behavioral shifts of instructors in their responsibilities to teach approximately 4800 undergraduate engineering students across three years (see Figure 1 for a visual overview of the project). Project activities will answer the following research questions at the intersection of learning analytics and faculty change to inform how to productively leverage institutional data to improve the STEM undergraduate education system, in particular at the stage during which students take foundational courses taught in large class sizes: 
RQ1: What data do STEM faculty teaching large foundational classes for undergraduate engineering identify as being useful so that they may enhance students' experiences and outcomes a) within the classes they teach, and b) across students' multiple large classes? RQ2: How can looking across data sets at different levels (i.e., within-course and across courses) change faculty members' attitudes or behaviors related to teaching undergraduate classes?

RQ3: How can looking across data sets at different levels produce insights related to systemic challenges that can be addressed by an academic program or institution?



Figure 1. Project at a glance: Investing in Instructors

\section{Progress of Work}

In Year 1 (see Figure 1) of this project, we focused on the experiences of instructors of foundational engineering courses usually taught in large class sizes. Project participants taught in 4 different departments at a large public research institution. We collected qualitative data (semistructured interviews, course-related documents) and quantitative data (student surveys, institution-provided transcript data, department-provided evaluation data) to answer our research questions.

The preliminary data were used as a baseline to further refine data collection protocols, identify data that faculty consider meaningful and useful, and consider ways of productively leveraging data to improve the learning experience in large foundational engineering courses. 
Participant interviews. We conducted semi-structured interviews with 8 instructors participating in Year 1, before the start of the Fall 2017 semester. The interviews, which lasted from approximately 40 minutes to one hour, were transcribed using a professional transcription service and analyzed using an online qualitative analysis software (www.dedoose.com).

Preliminary analysis on interview data was conducted concurrently by two members of the research team, in keeping with triangulation techniques [12]. First-cycle coding yielded 110 codes clustered around 17 themes, using a priori, in vivo, and descriptive coding techniques [13], [14]. The codebook was built iteratively, with the two investigators comparing coding decisions and making adjustments to code descriptions and clustering as part of the coding process. Themes included instructors' beliefs about students, challenges associated with the large class learning environment, and current practices and aspirations related to instructional processes and resources.

The participants were asked to identify data and resources that would help them facilitate the learning process more effectively. Based on participant responses, the most commonlyarticulated aspiration is for interaction and collaboration with instructors from other departments. Participants sought opportunities to interact and collaborate with instructors from other departments who: 1) teach pre-requisite or prior courses that prepare students for the course/s that they are currently teaching, and 2) teach succeeding courses where the knowledge and skills gained in the course/s the participant is currently teaching are important and useful.

For example, a participant who teaches mathematics shared: "As a resource that I would like, like from the department, from the university, is maybe to afford us some time to connect with colleagues outside of the department when we're teaching a class that services other departments, to get some input from those departments on why their students are in my room."

An instructor who teaches engineering mechanics courses expressed similar sentiments: "The other thing that we don't really do and we haven't done this anywhere that I've worked and it would be really nice if we could is get these instructors of different courses together to talk about exactly what to cover, to even just look over the syllabus. What's covered, what they expect their students to know, and what they don't expect their students to know as well." This participant also shared: "For all I know, whoever teaches next semester's course that follows [course], they might expect that their students understand one topic really very well and students are coming in with no idea how to do it because I don't spend any time on it because I don't think it's that important." 
These statements are further supported by responses that talk about seeking discipline-specific applications of course concepts from participants teaching in non-engineering departments, such as: "I also want to have the material I'm teaching somehow relevant to them, and that's something that I struggle with a little bit, because I don't have the engineering side."

Participant responses articulated data and resources that faculty consider valuable to the decision-making processes that shape the student learning experience in large foundational engineering courses. The information shared by our participants will allow us to identify strategies and facilitate opportunities to provide the instructors with the data that they need. Findings from the pre-semester interviews will inform activities and serve as catalyst for conversations during the Large Foundational Courses Summit, scheduled for Summer of Year 2.

Institutional and departmental data. We were provided access to institutional transcript data, from the Fall 2009 semester onwards. This data includes demographic information (e.g., gender, major), admissions data (e.g., SAT scores, high school GPA), and institutional academic information (e.g., course grades, cumulative GPA). The institutional transcript data provides such information as students' course-taking histories, major switching, degrees earned, and precollege characteristics.

Participating departments provided access to data (e.g., departmental survey data) that may be merged with the institutional transcript data. Analysis of institutional and departmental data is ongoing. Initial findings on quantitative analysis performed on this data will be presented and help guide discussions during the Large Foundational Courses Summit.

Student Surveys. Surveys focused on students' study habits were administered to students in classes taught by project participants from one participating department. The survey asked students to indicate the amount of time they spent engaging with course material as they prepared for a high-stakes test. The instrument was adapted from a previous study that explored learner engagement in a statics course conducted by some members of the project team [15], [16].

Data collection was conducted through paper surveys included as an insert in a high-stakes test. A pilot run was conducted during the Fall 2017 semester; student responses and feedback from the instructor-participants were used to make adjustments to the survey. An updated version of the survey is being administered during the Spring 2018 semester.

\section{Next steps}

Year 2 will begin with the $1^{\text {st }}$ Large Foundational Courses Summit. Participants to the first summit will include the first cohort of instructors who participated in the Year 1 activities. 
Preliminary findings from data collection and analysis activities conducted during Year 1 will be presented and will be used as basis for action plans that will be collaboratively prepared by the participants and the project team. A report will be generated at the end of the Summit, for distribution to the participants' departments and college-level administrators.

The second cohort of instructors will be recruited. Instructors from departments not currently participating in the project will be considered; identification of these departments will be part of the Year 2 Summit.

\section{References}

[1] C. Mulryan-Kyne, "Teaching large classes at college and university level: challenges and opportunities," Teach. High. Educ., vol. 15, no. 2, pp. 175-185, Apr. 2010.

[2] National Science Board, "Science and Engineering Indicators 2014," National Science Foundation, 2014.

[3] J. Cuseo, "The empirical case against large class size: adverse effects on the teaching, learning, and retention of first-year students," J. Fac. Dev., vol. 21, no. 1, pp. 5-21, 2007.

[4] E. Carbone, "Students Behaving Badly in Large Classes," New Dir. Teach. Learn., vol. 1999, no. 77, pp. 35-43, 1999.

[5] E. Carbone and J. Greenberg, "Teaching Large Classes: Unpacking the Problem and Responding Creatively," in To Improve the Academy, vol. 17, M. Kaplan, Ed. Stillwater, OK: New Forums Press and the Professional and Organizational Development Network in Higher Education, 1998, pp. 311-326.

[6] E. Seymour and N. M. Hewitt, Talking about leaving: why undergraduates leave the sciences. Westview Press, 1997.

[7] J. C. Chen, D. C. Whittinghill, and J. A. Kadlowec, "Classes That Click: Fast, Rich Feedback to Enhance Student Learning and Satisfaction," J. Eng. Educ., vol. 99, no. 2, pp. 159168, Apr. 2010.

[8] J. Gainen, "Barriers to success in quantitative gatekeeper courses," New Dir. Teach. Learn., vol. 1995, no. 61, pp. 5-14, 1995.

[9] L. R. Lattuca and J. S. Stark, Shaping the college curriculum: academic plans in context, 2nd ed. San Francisco, CA: Jossey-Bass, 2009.

[10] J. Grohs, G. Young, M. Soledad, and D. Knight, "Leveraging local data for reflective teaching in large classes," Innov. Educ. Teach. Int., vol. 0, no. 0, pp. 1-11, Feb. 2018.

[11] M. Borrego and C. Henderson, "Increasing the use of evidence-based teaching in STEM higher education: A comparison of eight change strategies," J. Eng. Educ., vol. 103, no. 2, pp. 220-252, 2014.

[12] J. A. Leydens, B. M. Moskal, and M. J. Pavelich, "Qualitative Methods Used in the Assessment of Engineering Education," J. Eng. Educ. Wash., vol. 93, no. 1, p. 65+, Jan. 2004.

[13] J. Saldana, The Coding Manual for Qualitative Researchers. SAGE Publications, 2009.

[14] M. B. Miles, A. M. Huberman, and J. Saldaña, Qualitative data analysis: A methods sourcebook, 3rd ed. Thousand Oaks, California: SAGE Publications, Incorporated, 2013.

[15] J. Grohs, T. Kinoshita, B. Novoselich, and D. Knight, "Exploring Learner Engagement and Achievement in Large Undergraduate Engineering Mechanics Courses," 2015, p. 26.729.126.729.11. 
[16] J. R. Grohs, D. B. Knight, G. D. Young, and M. M. Soledad, "Exploring Academic Performance Paths and Student Learning Strategies in a Large Foundational Engineering Course," Int. J. Educ. Math. Sci. Technol., in press. 